\title{
In vivo vs. ex vivo magnetic resonance imaging in mice
}

\section{Allan MacKenzie-Graham* \\ Multiple Sclerosis Program, Department of Neurology, University of California Los Angeles, Los Angeles, CA, USA \\ ${ }^{*}$ Correspondence: amg@ucla.edu}

\section{A commentary on}

Wanted dead or alive? The tradeoff between in vivo vs. ex vivo MR brain imaging in the mouse

by Lerch, J. P., Gazdzinski, L., Germann, J., Sled, J. G., Henkelman, R. M., and Nieman, B. J. (2012). Front. Neuroinform. 6:6. doi: 10.3389/fninf.2012.00006

The use of high-resolution MRI for the evaluation of structural changes in the mouse brain is rapidly gaining favor with researchers. The decision to use either in vivo or ex vivo imaging is often a practical one. For example, technical limitations, such as the availability of appropriate equipment to image in vivo, may force an investigator to use ex vivo imaging. Conversely, animal availability may limit the number of samples used at each time-point in an ex vivo experimental design, but not affect an in vivo design. But what if we were not limited by these considerations? What if we could decide which approach to take based entirely on which approach would yield the best data?

In "Wanted dead or alive? The tradeoff between in vivo vs. ex vivo MR brain imaging in the mouse" presented by Lerch et al. (2012), this is exactly what is considered. Ex vivo imaging benefits from greater resolution and sensitivity due to the lack of constraints on imaging time, the use of tighter fitting coils, high concentration contrast agents, and a lack of movement artifacts. In vivo imaging allows for the longitudinal analysis of structural change, a benefit that can not be underestimated. Longitudinal studies lend themselves to other forms of statistical analysis, such as repeated measures ANOVAs, which can increase the statistical power of the studies. But beyond the obvious differences, how sensitive are these MRI measures to structural changes on the order of $5 \%$ of the total volume of a neuroanatomical structure? The authors describe a series of statistical analyses (based on imaging data they acquired) used to evaluate the tradeoffs between the use of in vivo (longitudinal) analysis and ex vivo (cross sectional) analysis.

For a remarkably complex problem, the results are surprisingly straightforward. Firstly, ex vivo imaging is more precise than in vivo imaging. In cases where precise timecourse data is not required, ex vivo imaging provides better results. This is due to the lower within subject variability inherent to ex vivo imaging (higher resolution, no movement). However, if changes in absolute volumes or rates of change are required, in vivo imaging provides better information. Interestingly, in longitudinal experiments, the addition of more subjects, rather than more time-points, increases statistical power more rapidly.
Secondly, the kind of analysis used matters. Spatial normalization to an unbiased consensus average, correcting for gross differences in brain size, decreases the population standard deviation markedly (by approximately 50\%). Before spatial normalization, population standard deviations are about the same size as the effects to be observed. Clearly, spatial normalization is a crucial step in any form of volumetry.

As in vivo imaging equipment and expertise become more common, the decision between the use of in vivo or ex vivo imaging begins to boil down to choosing the best tool for the job; ex vivo for precision, in vivo for time-course, and spatial normalization for all.

\section{REFERENCE}

Lerch, J. P., Gazdzinski, L., Germann, J., Sled, J. G., Henkelman, R. M., and Nieman, B. J. (2012). Wanted dead or alive? The tradeoff between in-vivo versus ex-vivo MR brain imaging in the mouse. Front. Neuroinform. 6:6. doi: 10.3389/fninf.2012.00006

Received: 23 April 2012; accepted: 26 April 2012; published online: 17 May 2012.

Citation: MacKenzie-Graham A (2012) In vivo vs. ex vivo magnetic resonance imaging in mice. Front. Neuroinform. 6:19. doi: 10.3389/fninf.2012.00019

Copyright (c) 2012 MacKenzie-Graham. This is an openaccess article distributed under the terms of the Creative Commons Attribution Non Commercial License, which permits non-commercial use, distribution, and reproduction in other forums, provided the original authors and source are credited. 